\title{
High-intensity beam profile measurement using a gas sheet monitor by beam induced fluorescence detection
}

\author{
Ippei Yamada $\odot^{* \dagger \dagger}$ and Motoi Wada $\odot$ \\ Graduate School of Science and Engineering, Doshisha University, 1-3, Tatara-miyakodani, \\ Kyotanabe, Kyoto, 610-0321, Japan \\ Katsuhiro Moriya, Junichiro Kamiya, Pranab Kumar Saha๑, and Michikazu Kinsho \\ J-PARC Center (Japan Atomic Energy Agency), 2-4, Shirakata, Tokai-mura, \\ Naka-gun, Ibaraki, 319-1195, Japan
}

(Received 28 October 2020; accepted 22 March 2021; published 8 April 2021)

\begin{abstract}
A transverse beam profile monitor that visualizes a two-dimensional beam-induced fluorescent image was developed. The monitor employs a sheet-shaped gas flow formed by a technique of rarefied gas dynamics. A simplified analysis method was developed to reconstruct the beam intensity profile from the obtained image. The developed profile monitor and the analysis method were applied to measure the J-PARC $3 \mathrm{MeV} \mathrm{H}^{-}$beam profile. The root mean square values of the profiles were consistent with the ones obtained by a wire-scanning-type beam profile monitor. The beam loss due to the gas sheet injection was measured as a beam-current reduction. The amount of the beam current decreased in proportion to the gas sheet flux and the reduction ranged from 0.004 to $2.5 \%$. The assembled system was capable of reconstructing a beam profile from a single shot beam pulse $\left(1.7 \times 10^{13}\right.$ protons in $\left.50 \mu \mathrm{s}\right)$.
\end{abstract}

DOI: 10.1103/PhysRevAccelBeams.24.042801

\section{INTRODUCTION}

Measurements of high-intensity high-energy beams in a particle accelerator often cause losses of beams and activations of nuclear radiation of the accelerator system. A beam diagnostic system that minimizes the beam loss is to be realized for monitoring the beam operation of a highintensity high-energy accelerator. In J-PARC Linac, wire scanner monitors are utilized to measure the beam profile [1]. However, the beam pulse length must be shortened compared with the standard length because the wire breaks by the energy deposited from the beam particles. We are developing a nondestructive beam profile monitor system using a gas sheet to reduce the beam loss and improve the quality of the beam measurement.

Nondestructive beam profile monitors that detect charged particles, such as ions and electrons, or photons produced by beam-gas interaction are being developed in many research institutes. A monitor detecting charged particles has an advantage in the signal intensity compared with a monitor detecting photons. An important issue of the

\footnotetext{
*ip_yamada@icloud.com

Also J-PARC Center (JAEA).
}

Published by the American Physical Society under the terms of the Creative Commons Attribution 4.0 International license. Further distribution of this work must maintain attribution to the author(s) and the published article's title, journal citation, and DOI. charged-particle-detection monitor is the error in the measured beam profile due to different reasons: a wakefield of the beam, Coulomb interaction among the produced particles, and inhomogeneity of the electric field for collecting the produced particles [2]. Hochadel et al. [3] and Harada et al. [4] developed the multielectrode system for producing a uniform electric field to avoid the distortion of the measured beam profile. Vilsmeier et al. [2] and Bartkoski et al. [5] utilized the electrons guided by a magnetic field.

On the other hand, photon detection solves the issue of the profile distortion caused by the ambient electromagnetic field while it sacrifices the signal intensity [6-8]. Photon emission cross sections are usually smaller than ionization cross sections. In the case of $3 \mathrm{MeV}$ proton incident into $\mathrm{N}_{2}$ gas, the total ionization cross section is $6 \times 10^{-17} \mathrm{~cm}^{2}$ [9] compared to the total photon emission cross section of $1.2 \times$ $10^{-17} \mathrm{~cm}^{2}$ [10]. While the guiding electromagnetic field enables efficient collection of the charged particles, optical lenses can gather only $10^{-2}-10^{-4}$ of the total produced photons due to the solid-angle limitation.

Monitors injecting pencil- or sheet-shaped gas jet have been developed to increase the signal intensity [11-14]. Zhang et al. [11] and Tzoganis et al. [13] obtained a twodimensional beam profile using a supersonic sheet-shaped gas jet in the Cockcroft Institute. While the gas sheet jet is formed based on continuum (fluid) mechanics, a sheetshaped locally concentrated gas flow can be realized also 


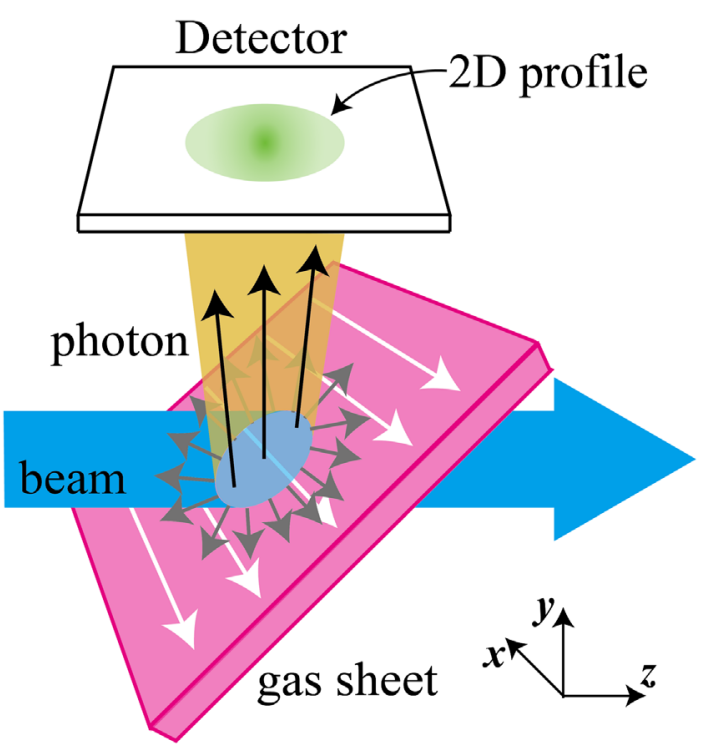

FIG. 1. A schematic diagram of a gas sheet beam profile monitor. The density distribution of the injected gas is concentrated like a thin sheet to enable measurements of the transverse beam profile in two dimensions without significant increase of the beam line gas pressure.

based on rarefied gas dynamics [15]. We have been developing the gas sheet monitor shown in Fig. 1 that forms a thin sheet of gas using the technique of rarefied gas dynamics. Advantages of our approach are the simplicity of the beam profile monitor structure and the gas flow simulation models and the wide pressure range of the gas sheet generation. For sheet formation of a fluid mechanics gas jet, the jet is formed by an adiabatic expansion around atmospheric pressure. Multistep pumping is required to form the pencil- or sheet-shaped gas jet and to decrease the gas pressure in the chambers from atmospheric pressure to ultrahigh vacuum. The gas flow simulation model is complex due to such a wide pressure range. The gas-jet-type monitor developed in the Cockcroft Institute [11-13] consists of six chambers including three prepumping chambers. The gas density of the jet reached $3 \times 10^{-5} \mathrm{~Pa}$ equivalent at $0.5 \mathrm{MPa}$ injection. In the Heavy Ion Medical Accelerator (HIMAC), Hashimoto et al. [14] developed an oxygen gas jet monitor consisting of five chambers including three prepumping chambers with focusing magnets. They realized the gas sheet jet of $1 \times 10^{-4} \mathrm{~Pa}$ at $0.6 \mathrm{MPa}$ injection. On the other hand, our gas sheet is formed not by an adiabatic expansion but by the thermal motion and reflections of the gas molecules on the gas sheet generator wall in the molecular flow condition where intermolecular collisions are negligible. A gas flow simulation for our monitor is easier because the gas flow from the generator entrance to a beam line can be modeled as a collisionless flow. The profile monitor system consists of two chambers including a prepumping chamber (see Secs. II B and IV B) because the operating gas pressure of the generator is low like medium or high vacuum to realize the collisionless condition. The generator being developed forms a gas sheet in a wide pressure range from a residual gas pressure level to $3 \times 10^{-3} \mathrm{~Pa}$ for the inlet pressure below $100 \mathrm{~Pa}$ [16].

We have been developing the gas sheet monitor separating into three components: (1) development of a gas sheet generator, (2) development of a calibration system of the monitor, and (3) test of beam profile measurements. As for part (1), a gas sheet generator is designed using a numerical simulation and tested to form a dense, homogeneous and thin sheet $[15,16]$. In part (2), the spatial distribution of the detection efficiency by taking the effect due to the gas density distribution into account has been quantified [17]. The precise evaluation of the detection efficiency spatial distribution is indispensable to reconstruct the beam profile from the obtained data by the gas sheet monitor. An electron beam narrow enough as compared to the ion beam whose spatial profile needs to be measured enables measurement of the detection efficiency spatial distribution. The electron beam induces local photon emissions from the gas sheet, and the distribution of the detection efficiency is measured by scanning the electron beam position on the gas sheet while monitoring the photon signal intensities. The spatial resolution of the gas sheet monitor is determined by comparing the signal detection position to the electron beam injection position. In part (3), the effectiveness of the gas sheet monitor is demonstrated for an actual beam acceleration system. We clarified several issues to be resolved for realizing the gas sheet monitor by the proof-of-principle test $[18,19]$. One of the issues is a proper analysis method for the beam profile reconstruction has not been developed yet; the obtained beam profiles were not evaluated quantitatively. Another issue is the beam loss induced by using the gas sheet monitor. While the gas sheet monitor has an advantage that the signal intensity increases due to injecting the gas compared with a residual gas monitor, the gas sheet monitor should induce a loss of the beam and this amount of the beam loss should be evaluated. In this paper, we report the effectiveness of the photon-detection-type gas sheet monitor based on the experiment results using the J-PARC $3 \mathrm{MeV} \mathrm{H}^{-}$beam from the following viewpoints: (i) reconstruction of a beam spatial distribution from a photon emission signal spatial distribution; (ii) comparison of a beam profile obtained by a gas sheet monitor using photons with a beam profile obtained by a wire scanner monitor; and (iii) discussion on merits and issues of the developed gas sheet profile monitor system.

\section{GAS SHEET MONITOR}

\section{A. Principle of beam profile measurement}

A local photon emission rate by beam-gas interaction is written as 


$$
n_{p}=\sigma n_{g} j / q
$$

where $n_{p}$ is the local photon emission rate, $\sigma$ is the emission cross section, $n_{g}$ is the local gas density, $j$ is the local beam current density, and $q$ is the charge of the beam particles.

Figure 2 shows the gas sheet monitor system that consists of a gas sheet injected along the $x$ axis and a CCD camera detecting light emissions along the $y$ axis. The figure also shows a beam passing through the gas sheet along the $z$ axis. The transverse beam intensity distribution (beam profile) corresponding to $j / q$, the gas density distribution corresponding to $n_{g}$, the detection efficiency distribution extended from $\sigma$, and the measured data of light intensity distribution corresponding to $n_{p}$ are defined as $f(x, y), n(x, y, z), \alpha(x, y, z)$, and $g(\tilde{x}, \tilde{y})$, respectively. The detection efficiency spatial distribution comprises the sensitivity distribution of the detector, the emission cross section, the detection solid angle, the transmittance of the view port and other photon losses including the ones at optical lenses. The coordinates with a tilde are assigned to the measured image. Parameters introduced in Fig. 2 have the following meanings:

$$
\begin{gathered}
p=y / \sin \theta-\tilde{y} / \cos \theta \\
q=y \cdot \cos \theta-\tilde{y} \cdot \sin \theta \\
\tilde{x}=x \\
\tilde{y}=y / \tan \theta(=z)
\end{gathered}
$$

with parameters $p$ and $q$ defined as axes perpendicular to the direction of the gas sheet flow.

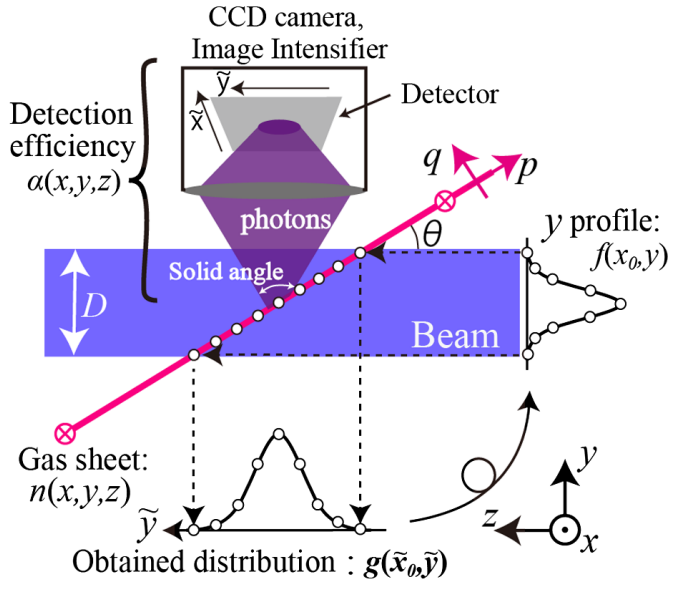

(a)
Considering the detector integrates photons along the $y$ axis, Eq. (1) can be extended as the following integral equation:

$$
g(\tilde{x}, \tilde{y})=\int_{D} \alpha(x, y, z) \cdot n(x, y, z) \cdot f(x, y) \mathrm{d} y,
$$

where the integration region $D$ is the typical beam size along the $y$ axis. The monitor's parameters $n(x, y, z)$, $\alpha(x, y, z)$ and the measured distribution $g(\tilde{x}, \tilde{y})$ give the beam profile for any gas sheet by solving the equation called the Fredholm (or the first kind integral) equation. To solve the equation consistently in an experimental measurement, the region $D$ can be replaced with a larger area, such as a beam pipe diameter. The integration vanishes where the beam distribution $f(x, y)=0$.

\section{B. Principle of gas sheet generator}

Formation of a gas sheet is based on rarefied gas dynamics [15]; the mean-free path of gas molecules is longer than the typical chamber size and interactions among molecules are negligible. The gas sheet generator shown in Fig. 3(a) is a long gas conduit of a thin cross section. In the case of the low generator inlet pressure where the mean-free path is longer than the conduit thickness, the motion of molecules in the conduit is determined by the thermal motion and reflections on the conduit wall. The molecules entering the conduit are aligned with a little emission angle spread by the reflections. In Fig. 3(a), the emission angle is described as the angle $\phi$ with respect to the $x$ axis. While there are molecules that return back to the inlet in the reflection process, the molecules that pass through the generator form a gas sheet. Collisions among the molecules forming a

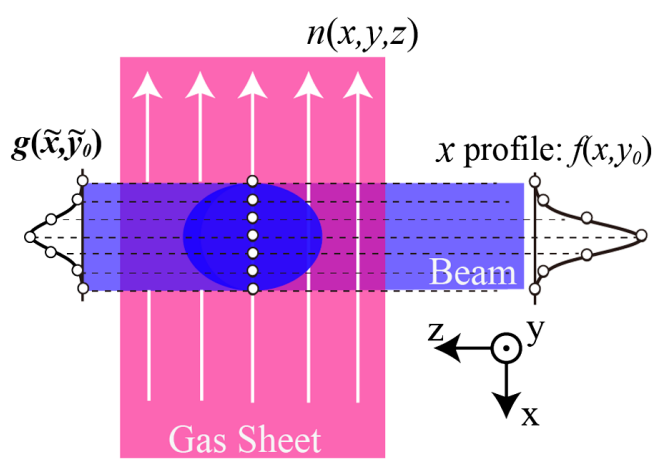

(b)

FIG. 2. The geometry of the gas sheet monitor and the beam: (a) the side view and (b) the top view. The camera captures the top view image of the photon distribution, $g(\tilde{x}, \tilde{y})$, produced by beam-gas interaction. The transverse beam intensity distribution, the gas density distribution, and the detection efficiency distribution are described as $f(x, y), n(x, y, z)$, and $\alpha(x, y, z)$, respectively. The typical beam size along $y$ axis is described as $D$. 


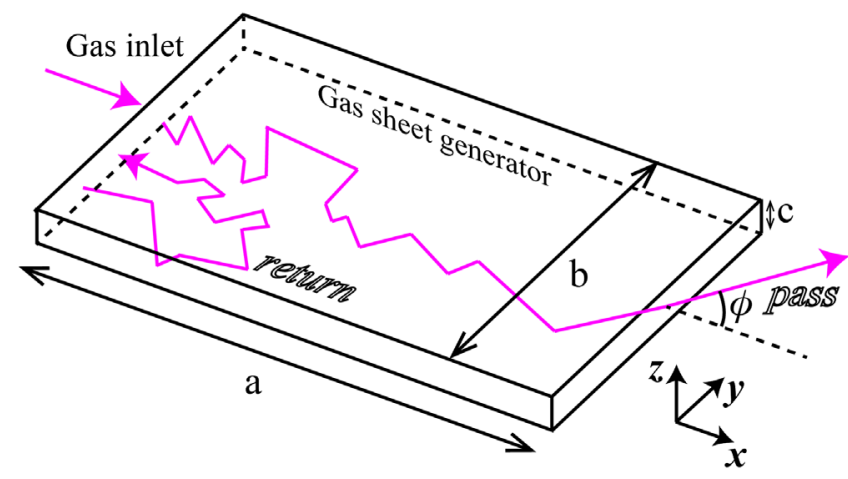

(a)

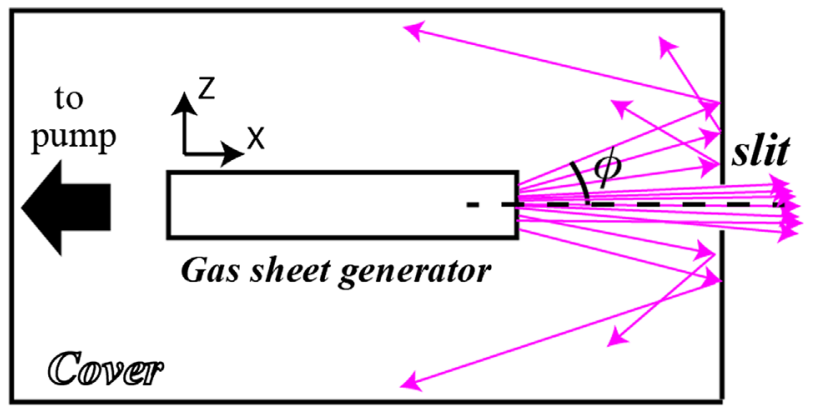

(b)

FIG. 3. Schematic diagrams of the formation principle of a gas sheet. (a) A thin and long gas conduit makes a gas sheet. (b) A cover slit eliminates the gas molecules that have large angle $\phi$.

sheet are also negligible because the gas sheet pressure (up to $10^{-3} \mathrm{~Pa}$ order) is low for a beam-pipe diameter (10 $\mathrm{mm}$ order).

In the case of the high generator inlet pressure where the mean-free path is shorter than the conduit thickness, the intermolecular collisions are not negligible in the conduit. The gas density distribution in this case is broader than the one in the collisionless case. The collisions become negligible after passing the generator exit because the gas sheet pressure decreases against the distance from the exit due to diffusion of the molecules when the pressure at the beam line is still low. A cover slit described in Fig. 3(b) surrounds the generator and limits the gas molecules that have large angle $\phi$ reaching the beam line to keep the beam line pressure low. The molecules reflected in the cover are eliminated by a vacuum pump attached to the cover. Though the gas spatial density distribution at high inlet pressure differs from the one at low inlet pressure, the gas density distribution keeps a sheet shape.

\section{BEAM PROFILE ANALYSIS METHOD}

In Sec. II A, we described that the integral equation [Eq. (6)] gives a beam profile from a measured photon distribution. Solving the equation needs numerical analysis in multiple precision, such as 1,000 digits [20], and the calculation needs a long time. In addition, analysis in high spatial resolution requires even a longer time because the number of data segments determines the calculation precision. A high speed analysis method based on proper approximation is desirable for implementing the beam monitor system to an accelerator. The following analysis method was developed.

The measured photon distribution well agrees to the actual beam profile when the gas density distribution is uniform and thin. For simplicity, the gas density distribution and the detection efficiency distribution are assumed to be ideal as follows:

$$
\begin{gathered}
n(x, y, z) \rightarrow n(x, p, q)= \begin{cases}1 & (\text { for } q=0, \text { any } x, p) \\
0 & (\text { otherwise })\end{cases} \\
\alpha(x, y, z)=1 \text { for any } x, y, z .
\end{gathered}
$$

In this assumption, the right-hand side of Eq. (6) vanishes except $q=0$. As a result, Eq. (6) takes the following form:

$$
g(\tilde{x}, \tilde{y})=f(x, y)
$$

with

$$
\begin{aligned}
& y=\tilde{y} \cdot \tan \theta \\
& x=\tilde{x} .
\end{aligned}
$$

These mean the measured photon distribution $g(\tilde{x}, \tilde{y})$ directly corresponds to the beam profile $f(x, y)$ at the coordinate transformations of Eq. (10): $g(x, y)=f(x, y)$. Equation (9) is valid including some possible error when the gas distribution can be regarded as uniform and thin across the main part of the beam.

We evaluated the error of the photon distribution $g(\tilde{x}, \tilde{y})$ against the beam profile $f(x, y)$ from the relation between the gas density distribution and the beam profile. Equation (6) constructs a photon distribution with an assumed gas density distribution and an assumed beam profile. The beam profile assumed in this study is approximated by a Gaussian distribution:

$$
f(x, y)=f_{0} \exp \left(-\frac{x^{2}}{2 \sigma_{x}^{2}}-\frac{y^{2}}{2 \sigma_{y}^{2}}\right) .
$$

The gas distribution cannot be approximated as a Gaussian distribution when collisions among molecules are negligible. However, for simplicity, we employ a Gaussian distribution with a constant term $n_{b}$ corresponding to the uniform background gas density:

$$
n(x, p, q)=n_{0} \exp \left(-\frac{q^{2}}{2 \sigma_{n}^{2}}\right)+n_{b} .
$$


The detection efficiency distribution is approximated as Eq. (8).

The response of the photon signal distribution along the detector axis ( $y$ axis) against variation of the gas sheet thickness $\sigma_{n}$ differs from the one along the other axis ( $x$ axis). We separate $g(x, y)$ to $g\left(x_{0}, y\right)$ and $g\left(x, y_{0}\right)$ and discuss the error caused by the choice of the parameters of the gas distribution, $\sigma_{n}, n_{0}$, and $n_{b}$ where $x_{0}$ and $y_{0}$ are constant positions.

\section{A. The error of $g\left(x_{0}, y\right)$}

The constant $n_{b}$ of the gas distribution in Eq. (12) forms the base part of the photon signal distribution $g\left(x_{0}, y\right)$ since the beam passes the gas sheet along the $\tilde{y}$ axis. The base part that does not have information of the beam profile $f(x, y)$ can be ignored. However, the base part intensity plays an important role to determine a dynamic range of profile measurement which is defined as a ratio of the peak and the base intensity of the photon distribution. If the base part is absent, the dynamic range of profile measurement corresponds to the one of the photon detector. This effect does not appear in $g\left(x, y_{0}\right)$ and will be further discussed in Sec. VI.

A root mean square (rms) value is employed to evaluate the error of the photon signal distribution $g\left(x_{0}, y\right)$ against the beam profile $f\left(x_{0}, y\right)$ quantitatively. The expected value of $g\left(x_{0}, y\right)$ is described as $\langle y\rangle$ and the rms value is written as follows:

$$
\mathrm{rms}=\sqrt{\frac{\sum_{i} g\left(x_{0}, y_{i}\right)\left(y_{i}-\langle y\rangle\right)^{2}}{\sum_{i} g\left(x_{0}, y_{i}\right)}} .
$$

In the case of a Gaussian distribution, an rms value equals the deviation $\sigma$. The rms value of $f\left(x_{0}, y\right)$ corresponds to $\sigma_{y}$. The error (Err) of the rms value is defined as follows:

$$
\text { Err }=\frac{\mathrm{rms}_{g_{y}}-\mathrm{rms}_{f_{y}}}{\mathrm{rms}_{f_{y}}}=\frac{\mathrm{rms}_{g_{y}}-\sigma_{y}}{\sigma_{y}},
$$

where $\mathrm{rms}_{g_{y}}$ indicates the rms value of $g\left(x_{0}, y\right)$. Figure 4 shows Err increases against increasing $\sigma_{n} / \sigma_{y}$. Accurate measurement of the beam profile along the $y$ axis using this approximate analysis method requires a thin gas sheet.

\section{B. The error of $\boldsymbol{g}\left(\boldsymbol{x}, \boldsymbol{y}_{\mathbf{0}}\right)$}

The distribution $g\left(x, y_{0}\right)$ gives an integrated photon signal distribution along the $y$ axis in the sheet thickness. While the photon signal distribution $g\left(x, y_{0}\right)$ corresponds to the beam profile of $f\left(x, y_{0}\right)$ for a small $\sigma_{n}, g\left(x, y_{0}\right)$ corresponds to a projected profile $f_{x}(x)=\int f(x, y) \mathrm{d} y$ for a big $\sigma_{n}$. The constant term $n_{b}$ of the gas density distribution works as the same for a big $\sigma_{n}$ and must not be ignored.

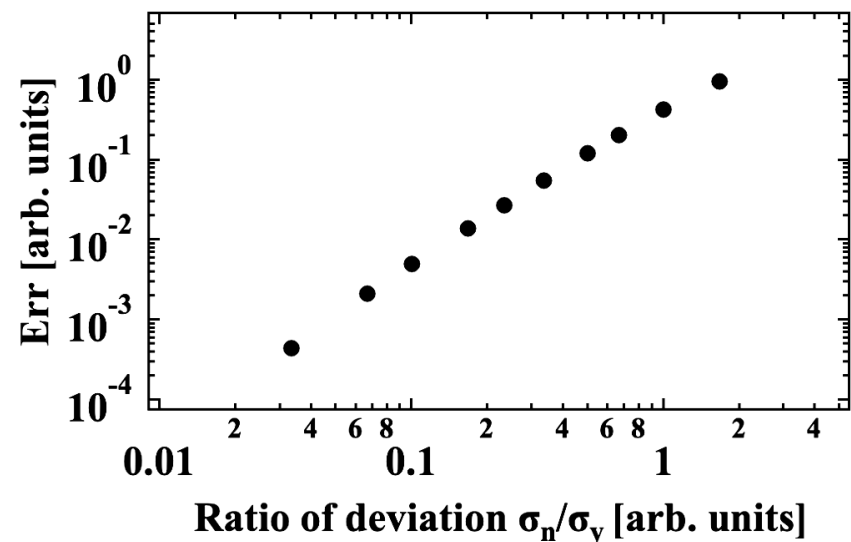

FIG. 4. The error between $g(0, y)$ and $f(0, y)$ against the thickness ratio of the gas sheet distribution and the beam distribution in the $y$ direction.

Here, when the beam profile is described as follows,

$$
f(x, y)=X(x) \cdot Y(y),
$$

the projected profile is proportional to a cross-sectional profile with a constant coefficient $C / c$ :

$$
\begin{gathered}
f\left(x, y_{0}\right)=X(x) \cdot Y\left(y_{0}\right)=c X(x) \\
f_{x}(x)=\int_{\ell} X(x) \cdot Y(y) \mathrm{d} y=C X(x) .
\end{gathered}
$$

The beam profile assumed in this study can be described by Eq. (15) as the product of Gaussian distributions in two independent coordinates. Hence, the measured distribution $g\left(x, y_{0}\right)$ is assumed to give the beam profile $f\left(x, y_{0}\right)\left[=f_{x}(x)\right]$.

\section{Summary and expected performance in J-PARC}

We summarize the features of the developed analysis method.

$y$ direction.-The beam profile can be obtained by Eqs. (9) and (10) with some error. The error of an obtained distribution increases as the gas sheet becomes thicker. The constant term of the gas distribution has no influence on the beam profile information but the term affects the dynamic range of profile measurement.

$x$ direction.-The beam spatial distribution is approximated to the photon distribution when the gas density distribution can be approximated to a simple Gaussian ignoring the precise spatial distribution.

Figure 5 shows the estimated photon signal distributions $g(x, 0)$ and $g(0, y)$ by Eq. (6) with the approximation employed for this study under the following parameters: (i) the gas density distribution: Gaussian distribution of $\sigma_{n}=2 \mathrm{~mm}$ (see Sec. IV B 5); (ii) the beam profile: Gaussian distributions of $\sigma_{x}=2 \mathrm{~mm}, \sigma_{y}=3 \mathrm{~mm}$. 


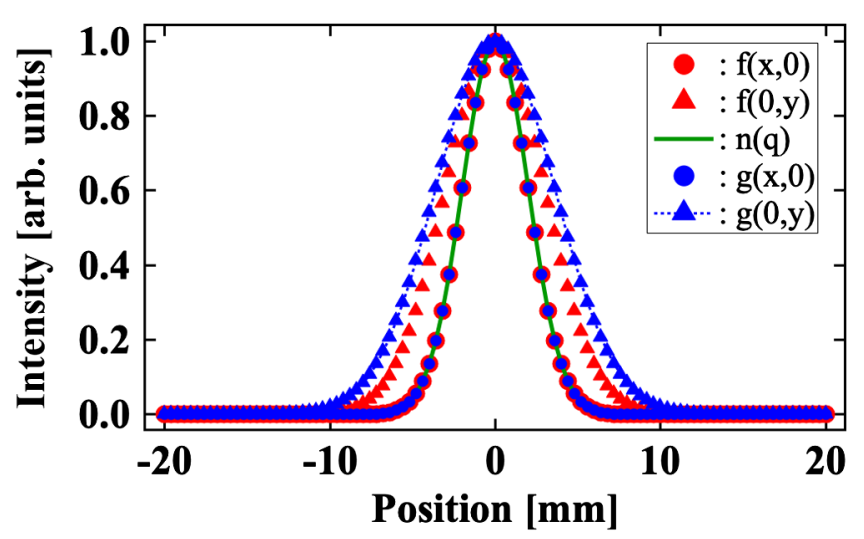

FIG. 5. Estimations of the beam profiles expected to be measured using the gas sheet monitor with typical parameters of the J-PARC beam profile and the gas density spatial distribution. The center position of the beam is $(x, y)=(0,0)$. The beam distribution is approximated as Gaussian distributions $\sigma_{x}=2 \mathrm{~mm}, \sigma_{y}=3 \mathrm{~mm}$, and the gas distribution is also approximated as a Gaussian distribution $\sigma_{n}=2 \mathrm{~mm}$.

The red circular and triangular plots describe the beam profiles along the $x$ and $y$ axes, respectively. The green line describes the gas density distribution along the $q$ axis that is perpendicular to the gas sheet plane. The blue circular and triangular plots describe the photon signal distributions expected to be measured with the beam and the gas spatial distributions. The photon signal distribution along the $x$ axis is expected to agree with the beam profile. For the $y$ direction, the photon distribution is broader than the beam profile by $20 \%$ on the rms value since $\sigma_{n} / \sigma_{y}=0.67$.

\section{EXPERIMENTAL SETUP}

\section{A. MEBT test stand of J-PARC}

Figure 6 shows a schematic diagram of the test stand of the J-PARC medium energy beam transport (MEBT) line $[21,22]$. The typical beam parameters of the test stand are described in Table I. The operation monitor system of the radio frequency quadrupole linac (RFQ) limits the gas

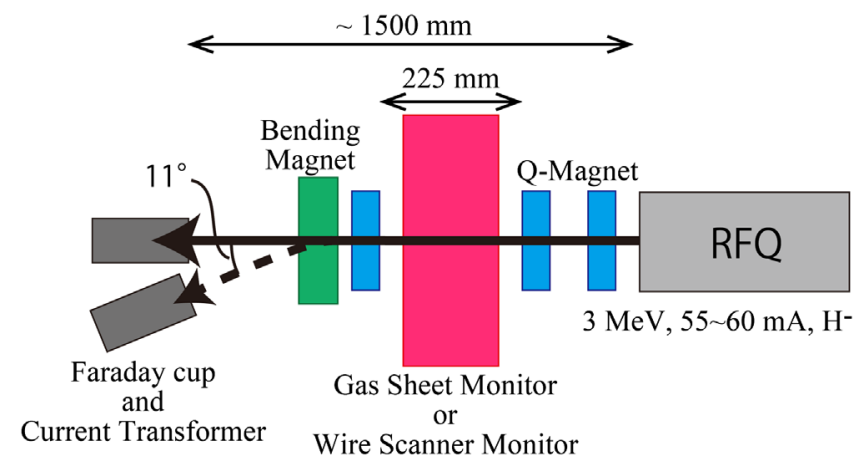

FIG. 6. The medium energy beam transport (MEBT) line test stand of the J-PARC Linac. The amount of the gas flux is limited to keep the gas pressure at the RFQ to be less than $5 \times 10^{-5} \mathrm{~Pa}$.
TABLE I. The typical parameters of J-PARC medium energy beam transport (MEBT) line test stand.

\begin{tabular}{lc}
\hline \hline Beam species & $\mathrm{H}^{-}$ \\
Beam energy & $3 \mathrm{MeV}$ \\
Resonant frequency (RFQ) & $324 \mathrm{MHz}$ \\
Peak beam current & $55-60 \mathrm{~mA}$ \\
Pulse length & $50 \mu \mathrm{s}$ \\
& $\left(1.7 \times 10^{13}\right.$ protons per pulse) \\
Repetition rate & $6 \mathrm{~Hz}$ \\
Transverse rms beam size & $2-3 \mathrm{~mm}$ \\
Transverse rms emittance & $0.3 \pi \mathrm{mm}$ mrad (normalized) \\
\hline \hline
\end{tabular}

injection flux not to exceed $5 \times 10^{-5} \mathrm{~Pa}$ of the gas pressure at the RFQ. A beam profile obtained by the gas sheet monitor was compared with a profile obtained by a wire scanner monitor placed at the same position of the MEBT line as the gas sheet monitor. A bending magnet below the gas sheet monitor separates the beam at 11 degrees. For a beam-loss measurement the $\mathrm{H}^{-}$current was measured by current transformers after bending the beam.

\section{B. Gas sheet monitor system}

The developed gas sheet monitor system is shown in Fig. 7. The monitor consists of a gas sheet generator which has a cuboid gas flow path of $a: b: c=$ $100 \mathrm{~mm}: 50 \mathrm{~mm}: 0.1 \mathrm{~mm}$ as described in Fig. 3(a), a cover chamber, a main chamber, vacuum pumps, and a photon detector. The gas sheet was injected continuously. The details are described as follows.

\section{Gas sheet generator and cover chamber}

Exhaust of sheet gas molecules is the most important technological challenge in developing a gas sheet monitor system. While the gas sheet generator aligns the momentum vectors of molecules, the molecules having large emission angles do not disappear due to the finite thickness of the gas flow path. These molecules should be pumped out before injection to a beam line to keep low beam line pressure. A $0.5 \mathrm{~mm} \times 50 \mathrm{~mm}$ rectangular slit with a cover chamber described in Fig. 3(b) was attached to $10 \mathrm{~mm}$ downstream from the exit of the gas sheet generator as shown in Fig. 8. Collisions among the gas molecules in the cover chamber $(0.1 \mathrm{~m}$ order $)$ are negligible for a pressure less than $10^{-2} \mathrm{~Pa}$ (mean-free path $=1 \mathrm{~m}$ ). The probability for a gas molecule passing the slit was calculated by MOLFLOW+ code [23], which led to the conductance of $15 \ell / \mathrm{s}$. A turbomolecular pump (TMP) that has a pumping speed of $420 \ell / \mathrm{s}$ (for $\mathrm{N}_{2}$ ) evacuates gas molecules in the cover chamber reflected at the slit.

\section{Main chamber}

The gas molecules forming the sheet at the beam line are pumped out by a cryopump and a TMP with the pumping 


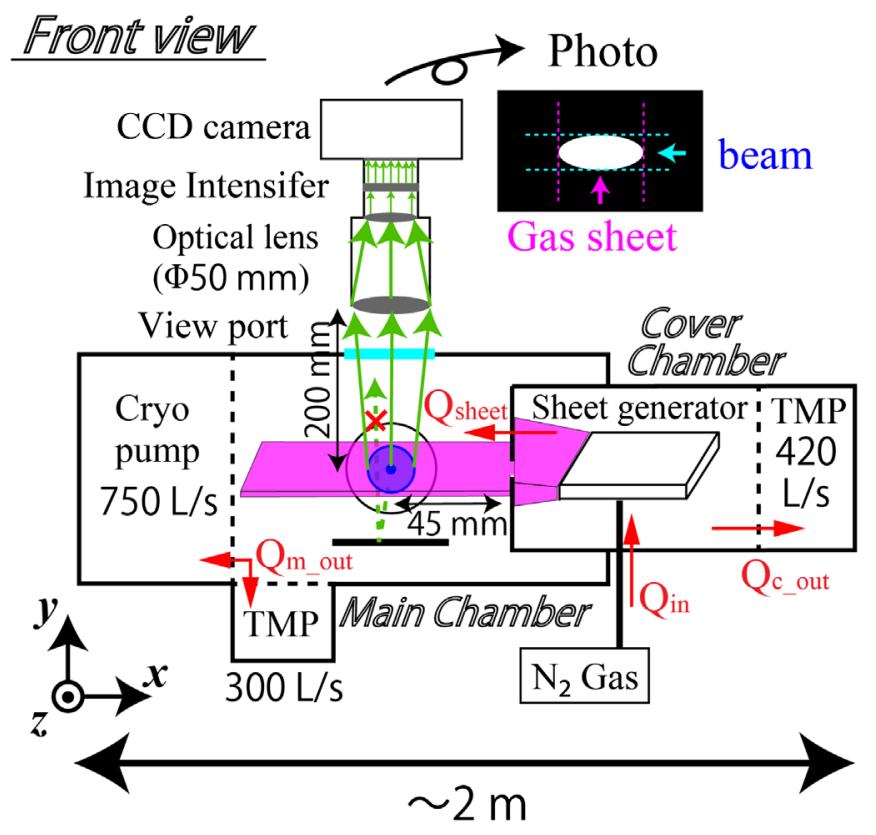

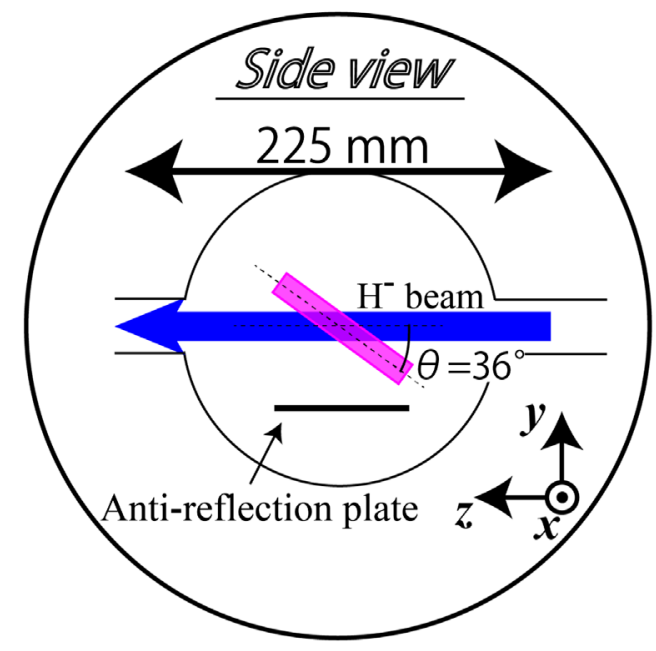

(b)

(a)

FIG. 7. The gas sheet monitor system: (a) the front view and (b) the side view. The system consists of a main chamber connected to a beam line, a gas sheet generator attached to a cover chamber that consists of a $0.5 \mathrm{~mm} \times 50 \mathrm{~mm}$ slit and a TMP, two vacuum pumps, and a photon detector system consisting of optical lenses, an image intensifier, a CCD camera, and an antireflection plate. The gas sheet tilts 36 degrees from the beam.

speed against $\mathrm{N}_{2}$ of 750 and $300 \ell / \mathrm{s}$, respectively. Figure 9 indicates the gas pressures at the main chamber, the pressure at the RFQ, the flux of the gas sheet $Q_{\text {sheet }}$, and the introduced gas flux to the generator $Q_{\text {in }}$ as a function of the inlet pressure of the gas sheet generator. These fluxes are calculated as follows:

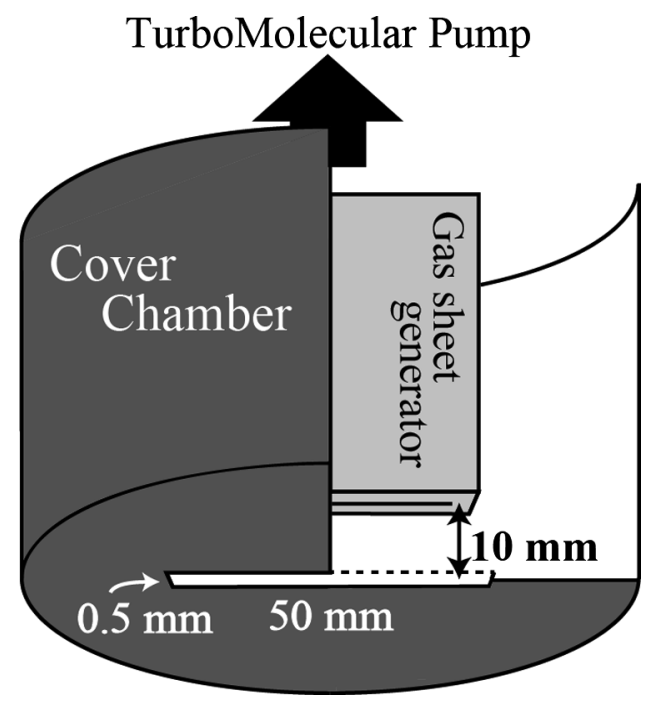

FIG. 8. The cover chamber and the gas sheet generator. The distance from the exit of the gas sheet generator to the slit is $10 \mathrm{~mm}$.

$$
\begin{gathered}
Q_{m_{-} \text {out }}, Q_{c_{-} \text {out }}=(\text { pumping speed }) \times(\text { pressure }) \\
Q_{\text {sheet }}=Q_{m_{-} \text {out }} \\
Q_{\text {in }}=Q_{\text {sheet }}+Q_{c_{\_} \text {out }} .
\end{gathered}
$$

The cover chamber pumped out around $90 \%$ of the injected gas as expected and the main chamber pumped out the rest which forms the gas sheet. The gas sheet introduction had little influence on the gas pressure of the RFQ (1 m upstream from the gas sheet monitor).

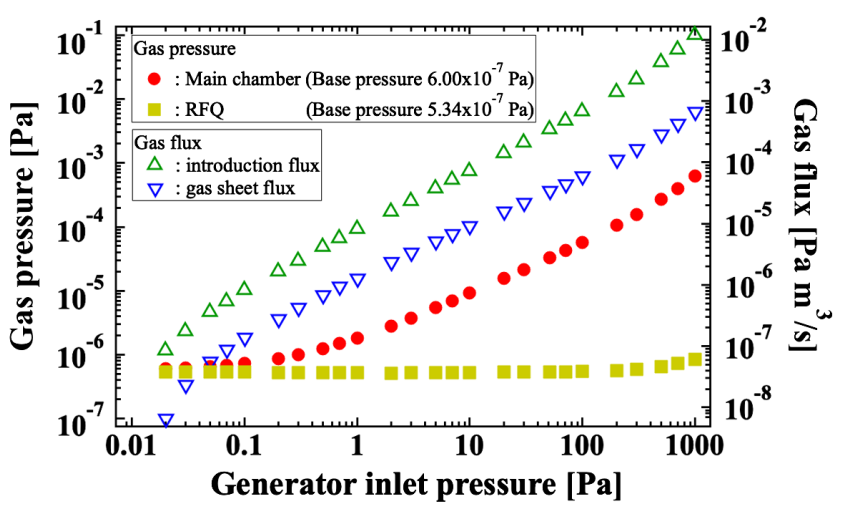

FIG. 9. The gas pressures at the main chamber, the pressure at the RFQ, the gas flux forming the gas sheet, and the introduced gas flux to the generator against the inlet gas pressure. 


\section{Photon detector}

The photon detector consists of a set of optical lenses

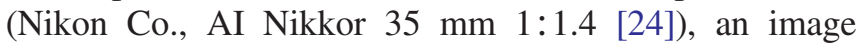
intensifier (Hamamatsu Photonics K.K., C9016-21 [25]), and a CCD camera (BITRAN Co., BU-66EM [26]). The image intensifier transforms photons to electrons and amplifies the electrons with a built-in multichannel plate. The electrons create an optical image by striking a phosphor screen. The CCD camera captures the image on the screen. The camera has $1920 \times 1080$ pixels of 16 bit CCD image sensors that are $5.5 \mu \mathrm{m}$ square each. One side of the sensor pixel on the images corresponds to $32 \mu \mathrm{m}$ at the position of the beam-gas-interaction point. An antireflection plate was placed to reduce the number of photons reflected on the chamber wall which blur the beam profile image. The distance from the beam-gas-interaction point to the optical lenses (50 mm of diameter) is $200 \mathrm{~mm}$. The detectable solid angle is $0.025 \mathrm{sr}(0.39 \%$ of $4 \pi)$.

\section{Gas species}

Nitrogen gas was employed as the injection gas species due to the large photon emission cross section. The total emission cross section of nitrogen is 3 times larger than the one of xenon or argon [10]. Excited $\mathrm{N}_{2}$ gas molecules live for $60 \mathrm{~ns}$ on average. The excited molecules can move from the beam-gas-interaction point during the lifetime. The distance of the particle movement should be estimated because the motion makes the beam profile image blurry. The main excitation process is expected as $B^{2} \Sigma_{u}{ }^{+} \rightarrow$ $X^{2} \Sigma_{g}^{+}$(the first negative band), and its main wavelengths are $391.4 \mathrm{~nm}, 427.8 \mathrm{~nm}:\left(v^{\prime}, v^{\prime \prime}\right)=(0,0),(0,1)$ [10]. The motion of molecules is estimated with an initial velocity and a Coulomb interaction.

(1) Initial velocity.-The thermal velocity and the momentum transfer during the collision contribute. DeLuca et al. measured momentum transfer is negligible compared with the thermal motion [27]. Xie et al. described excitation of electrons as using most of the energy which molecules obtain by the interaction [28]. The contribution of momentum transfer is ignored in this assumption. The gas sheet molecules are considered to be in a thermal equilibrium with the generator wall. The speed distribution function of molecules is assumed Maxwellian,

$$
f(v)=4 \pi v^{2}\left(\frac{m}{2 \pi k_{B} T}\right)^{3 / 2} \exp \left(-\frac{m v^{2}}{2 k_{B} T}\right),
$$

where $v$ is speed of molecules, $m$ is mass of molecules, $k_{B}$ is the Boltzmann constant, and $T$ is temperature of the wall. The beam profile blurring occurs not by the mean speed of gas molecules but by the speed deviation. In the case of $\mathrm{N}_{2}$ molecules and $300 \mathrm{~K}$, the deviation in the thermal speed is $205 \mathrm{~m} / \mathrm{s}$. The error due to the initial velocity is estimated as $205 \mathrm{~m} / \mathrm{s} \times 60 \mathrm{~ns}=12 \mu \mathrm{m}$.
(2) Coulomb interaction.-The beam interacts with the excited molecules electromagnetically because the main excited molecules are $\mathrm{N}_{2}{ }^{+}$. The J-PARC beam pulse consists of micropulses which include $10^{9}$ protons per micropulse. The electric field in transverse induced by a micropulse is expected as $2 \times 10^{5} \mathrm{kV} / \mathrm{m}$ at maximum. The duty of the micropulse in a beam pulse is 0.034 , and an averaging approximation leads the electric field of $2 \times 10^{5} \times 0.034 \mathrm{kV} / \mathrm{m}$. The equation of motion with the averaged electric field shows the excited molecules can move $46 \mu \mathrm{m}$ in $60 \mathrm{~ns}$.

The total motion is estimated as $\sqrt{12^{2}+46^{2}}=48 \mu \mathrm{m}$ in quadrature because the contributions do not always occur in the same direction. The effect due to the motion can be neglected because it is about the same to the spatial resolution of the photon detector $(32 \mu \mathrm{m})$.

\section{Detection efficiency distribution and gas density distribution}

The gas sheet monitor system constitutes a detection efficiency spatial distribution $\alpha(x, y, z)$ and a gas density spatial distribution $n(x, y, z)$ according to the inlet gas pressure of the generator. The product distribution $\alpha(x, y, z) \cdot n(x, y, z)$ at the inlet pressure of $100 \mathrm{~Pa}$ shown in Fig. 10 is quantified with an electron beam instead of a $\mathrm{H}^{-}$beam as described in Sec. I. The distribution mainly reflects the contribution of the gas sheet distribution along the $z$ axis. While the gas density distributions along the $p$ and $x$ axes are neglected, the gas distribution along the $q$ axis fits to a Gaussian distribution of $\sigma=1.96 \mathrm{~mm}$ by considering the gas sheet tilt. This result implies the gas flow model cannot be treated as completely collisionless for the inlet pressure of $100 \mathrm{~Pa}$ against the gas conduit thickness of $0.1 \mathrm{~mm}$. A thinner and denser gas sheet with the large pressure difference between the peak and the

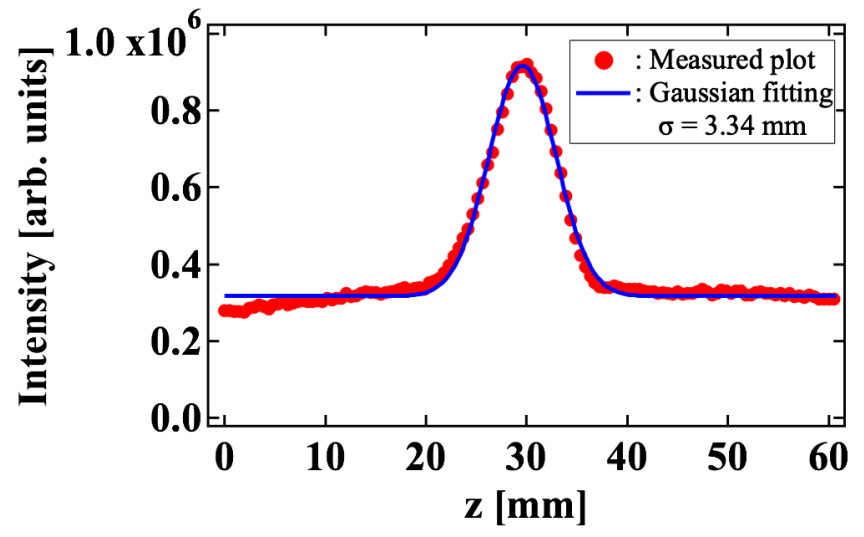

FIG. 10. An example of the product of the detection efficiency distribution and the gas density distribution along the $z$ axis at the center of the $\mathrm{H}^{-}$beam trajectory. The inlet pressure of the gas sheet generator is $100 \mathrm{~Pa}$. 


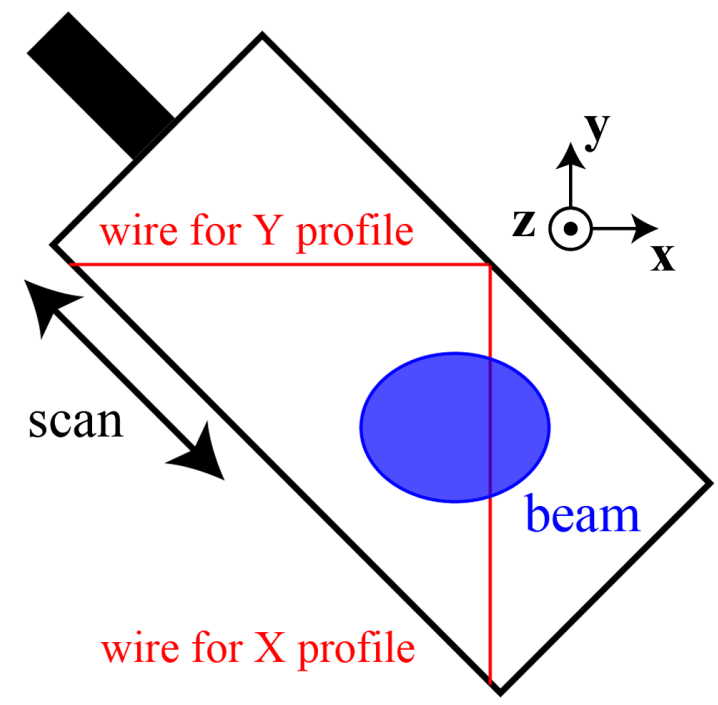

FIG. 11. A schematic diagram of a wire scanner monitor. The wire is made of carbon nanotubes and the wire diameter is $100 \mu \mathrm{m}$.

background such as more than 20 times is realized by a new gas sheet generator [16].

\section{Wire scanner monitor}

Figure 11 shows a schematic diagram of the wire scanner monitor used for the present experiment. A one-dimensional probe (wire) measures a projected beam profile. The wire is made of carbon nanotubes and the wire diameter is $100 \mu \mathrm{m}$. Each measurement point of the profile utilizes one pulse, and the spatial step of measurement is defined as $0.35 \mathrm{~mm}$ along the $x$ or the $y$ axis. Beam profile measurements along two axes need 150 pulses.

\section{V. $\mathrm{H}^{-}$BEAM DETECTION RESULTS}

\section{A. Beam profile measurement}

\section{Captured image}

An example of a CCD-camera-captured image is shown in Fig. 12. The measurement conditions were the inlet pressure of $100 \mathrm{~Pa}$ and $50 \mu \mathrm{s} \times 12,000$ pulse integration $\left(=2.0 \times 10^{17}\right.$ protons $)$. The gas sheet flowed toward $-\tilde{x}$ and the beam passed the sheet toward $-\tilde{y}$. The white horizontal line image indicates the beam-induced fluorescence against the background gas. The signal against the sheet core was detected in the center part. These two contributions to the signal appeared as expected in Sec. III A. The cross-sectional and projected luminous signal intensity distributions of Fig. 12 are shown in Fig. 13. The cross sections include the point giving the peak intensity $[(\tilde{x}, \tilde{y})=(20.0 \mathrm{~mm}, 31.5 \mathrm{~mm})$ that is common for the $\tilde{x}$ and $\tilde{y}$ profile. The intensities of Fig. 13 are normalized with the peak intensity.

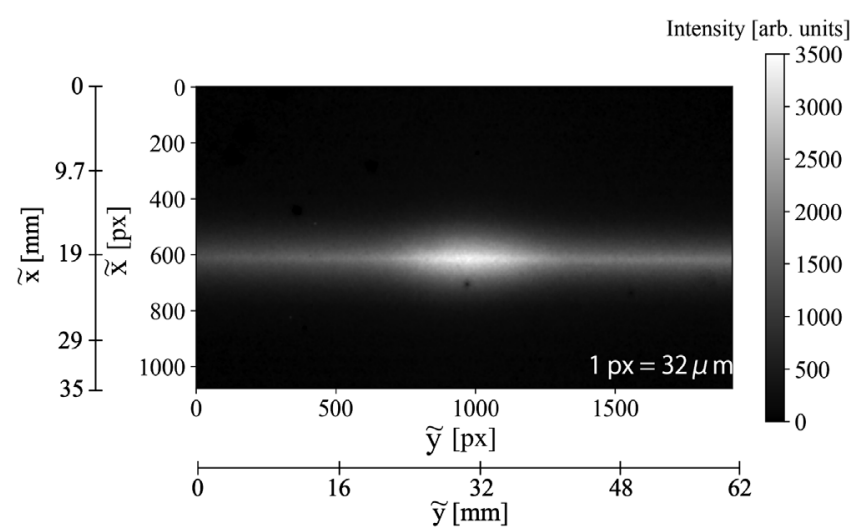

FIG. 12. An example of an image obtained by the gas sheet monitor. The white part is the photon signal. The geometry of the gas sheet and the $\mathrm{H}^{-}$beam is described in Fig. 7.

\section{Beam profile}

Beam profiles are reconstructed from the image by applying the approximate analysis method described in Sec. III. For the $x$ profile, the obtained signal distribution is described with the base part $B$ as follows:

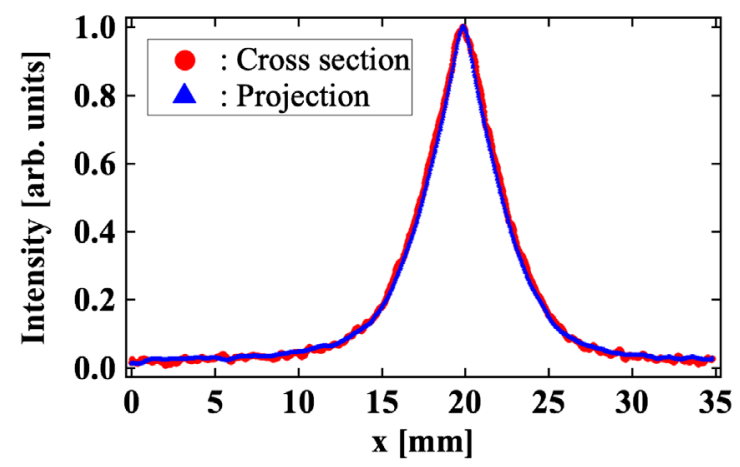

(a)

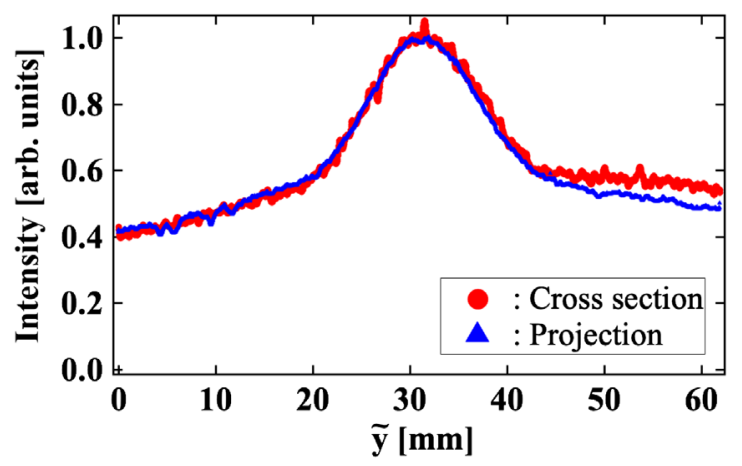

(b)

FIG. 13. The measured distributions $g(\tilde{x}, \tilde{y})$ at the inlet pressure of $100 \mathrm{~Pa}$ and $50 \mu \mathrm{s} \times 12,000$ pulse integration. (a) Vertical distributions of the luminous intensity corresponding to the beam profiles along the $x$ axis. (b) Horizontal distributions of the luminous intensity corresponding to the beam profiles along the $y$ axis. 


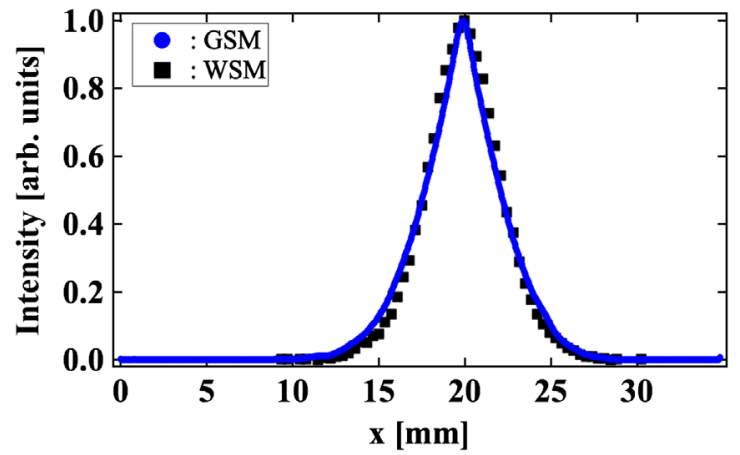

(a)

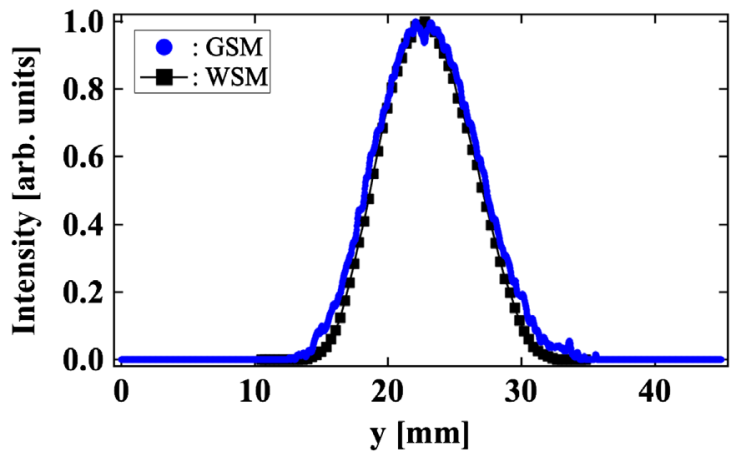

(b)

FIG. 14. The beam profiles obtained by the gas sheet monitor (GSM) are compared with those obtained by the wire scanner monitor (WSM): (a) the projected $x$ profiles and (b) the projected $y$ profiles.

$$
g_{\tilde{x}}(\tilde{x})=g_{x}(x)=f_{x}(x)+B .
$$

Hence, the signal corresponds to the beam profile by removing the base part $B$. The base part was caused by a read noise of the CCD camera and a beam-induced noise. The beam-induced noise is partly caused by the fluorescence from the beam dump placed $1 \mathrm{~m}$ downstream of the gas sheet monitor. The beam strikes the dump to heat it up to a temperature for emission of thermal radiation light. For the $y$ profile, the obtained signal distribution is described in the following form:

$$
g_{\tilde{y}}(\tilde{y})=f_{\tilde{y}}(\tilde{y})+B(\tilde{y}) .
$$

The background gas density distribution and nonuniformity of the detection efficiency caused the base function $B(\tilde{y})$. The base part which does not have beam profile information should be removed to evaluate the profile. The ends of the $y$ profile $f_{\tilde{y}}(\tilde{y})$ are found out from taking the derivative of the signal distribution of Fig. 13(b). The tail parts of the first derivative can be assumed as constant and the distribution slope changes at $\tilde{y}=17.3 \mathrm{~mm}, 49.1 \mathrm{~mm}$. These points are defined as the profile-end positions. For simplicity, $B(\tilde{y})$ is defined as a constant of 0.52 which is the intensity at the points and the entire signal is trimmed off
TABLE II. Comparison of the rms values obtained by the gas sheet monitor (GSM) and the wire scanner monitor (WSM).

\begin{tabular}{lccc}
\hline \hline & GSM $[\mathrm{mm}]$ & WSM $[\mathrm{mm}]$ & Difference \\
\hline$x$ profile & 2.38 & 2.26 & $5.31 \%$ \\
$y$ profile & 3.66 & 3.16 & $15.8 \%$ \\
\hline \hline
\end{tabular}

with this constant. Axis transformation by $y=\tilde{y} \cdot \tan \theta$ is also applied where $\theta=36$ degrees.

Figure 14 shows the $x$ and $y$ profiles measured by the gas sheet monitor and the wire scanner monitor. The $y$ profile measured by the gas sheet monitor is normalized again after trimming the base. Both $x$ and $y$ profiles measured by the gas sheet monitor well agree with those measured by the wire scanner monitor. The rms values are described in Table II as quantitative indices. The tendency of the differences in the rms values is consistent with the expected one in Sec. III.

\section{Two-dimensional mapping}

Agreement of the beam profiles measured by the gas sheet monitor and the wire scanner monitor indicates the developed approximate analysis method assuming an ideal gas density distribution can estimate the beam profile with reasonable accuracy. One of the advantages of the gas sheet monitor is its capability of reconstructing a two-dimensional beam profile. A mapping of the transverse beam profile which can be reconstructed by applying the analysis to all $\tilde{y}$ cross-sectional distributions of Fig. 12 is shown in Fig. 15. This two-dimensional profile includes the error estimated in Sec. III and Table II.

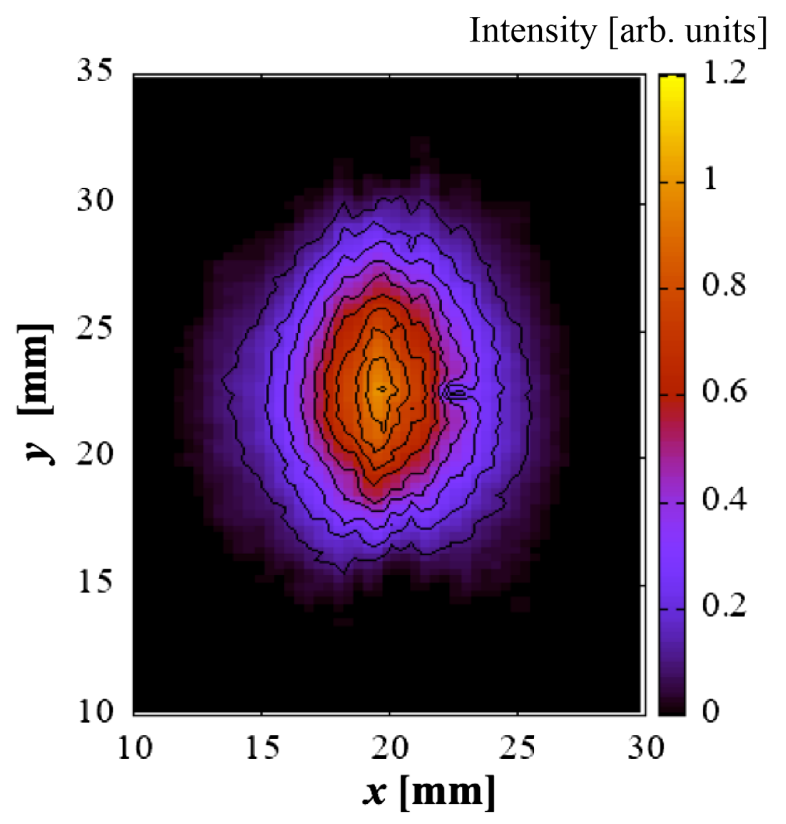

FIG. 15. A two-dimensional mapping of the transverse beam profile of Fig. 12. 


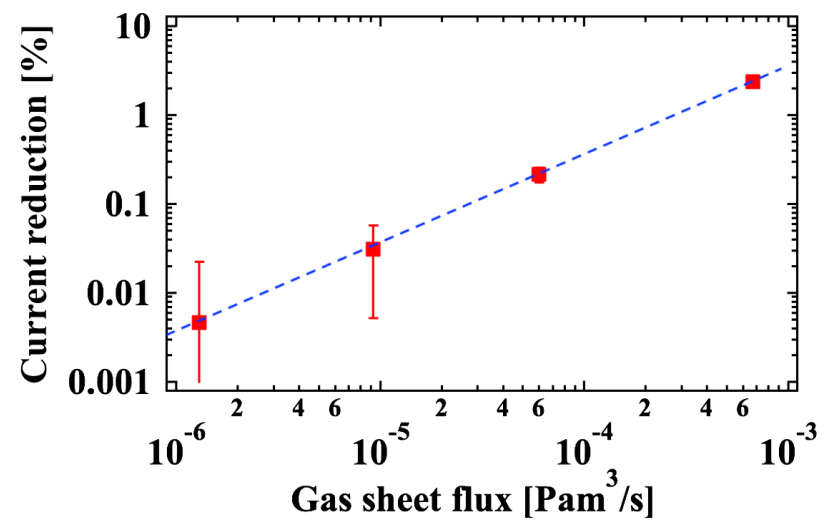

FIG. 16. The beam current reduction due to the gas injection measured by current transformers. The error bars describe the deviation of the current reduction. The blue broken line is the fitted line to a linear function.

\section{B. Beam loss due to the gas sheet monitor}

Gas injection causes a beam loss even if the mass density of the gas is far lower than the one of the wire. The beam loss induced by the gas sheet monitor was evaluated by the beam-current reduction. Current transformers measured the amount of the $\mathrm{H}^{-}$beam current after an 11 degree beam bending by a magnet installed downstream of the gas sheet monitor. Figure 16 indicates the beam-current reduction plotted as a function of the gas sheet flux. The current reduction rate increases in proportion to the gas sheet flux. At $6 \times 10^{-5} \mathrm{~Pa} \mathrm{~m}^{3} / \mathrm{s}$ flux which corresponds to the condition of Figs. 12-15, the $\mathrm{H}^{-}$beam current decreases by $0.21 \%$.

\section{DISCUSSION}

Compared with the wire scanner monitor, the gas sheet monitor system does not require pulse-to-pulse measurement by mechanically sweeping the position of the wire detectors; the measurement can be made with a single pulse. However, the signal intensity and the data quality must be improved by accumulating the number of pulses. Figure 17 shows a ten-pulse-integration beam profile measured by the gas sheet monitor at the inlet pressure of $100 \mathrm{~Pa}$ with the profile integrated over 12,000 pulses. In the $x$ direction, the ten-pulse profile can be recognized. In the $y$ direction, the ten-pulse profile is noisy and not clearly recognized. Moving average at $1 \mathrm{~mm}$ makes the distribution clear. A noise part of a signal-to-noise ratio $(\mathrm{S} / \mathrm{N})$ is defined as the deviation at the tail of the beam distribution where the distribution can be approximated as a constant. The $\mathrm{S} / \mathrm{N}$ for the $y$ profile [Fig. 17(b)] is smaller than the one for the $x$ profile [Fig. 17(a)] because of the reduction of the dynamic range for the $y$ profile described in Sec. III A. In the profile analysis process, subtraction of $B(\tilde{y})=0.52$ to extract the beam profile causes the signal intensity reduction to 0.48 , and the $\mathrm{S} / \mathrm{N}$ for the $y$ profile becomes small.

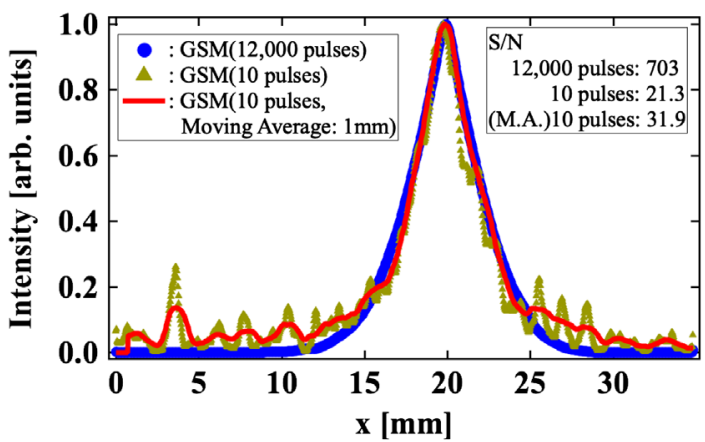

(a)

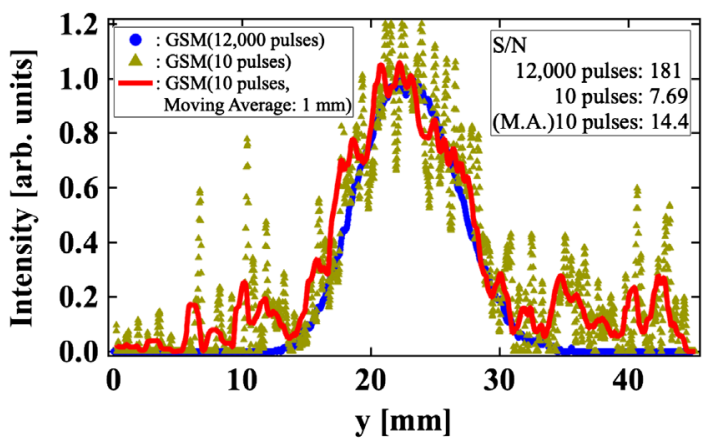

(b)

FIG. 17. The beam profiles obtained by the gas sheet monitor (GSM) integrating ten or 12,000 beam pulses: (a) the projected $x$ profiles and (b) the projected $y$ profiles. The $y$ profiles are normalized after subtraction of the base part.

The beam in the low energy range, such as $3 \mathrm{MeV}$, induces large energy transfer to the interaction material according to the Bethe-Bloch formula. An amount of charges in one pulse is limited when a wire scanner monitor measures the beam profile because the wire may be broken. In this study, the pulse length was shortened down to $50 \mu \mathrm{s}$ against the user operation parameter: $500 \mu \mathrm{s}$. On the other hand, high-intensity beam operation advantages the signal intensity for a gas sheet monitor. Figure 17 implies the gas sheet monitor can measure the $500 \mu$ s-beam profile by only one pulse. An increase of the gas sheet flux by 10 times also enables the one-pulse measurement for $50 \mu \mathrm{s}$ beam. Considering these two possibilities, the gas sheet monitor has a potential to measure time evolution of a beam spatial structure in a pulse.

\section{CONCLUSION}

A beam-induced fluorescence profile monitor using a gas sheet was developed. There were three development issues: establishment of a profile analysis method, quantitative evaluation of an obtained beam profile, and evaluation of beam loss due to gas injection. We obtained the following results for these issues.

(i) An approximate analysis method deduced from the Fredholm integral equation reconstructs the beam profile 
with reasonable accuracy. This approximate analysis method and the method solving the integral equation can be utilized for ordinary beam-induced fluorescence beam profile monitors. The latter method does not limit the gas density distribution to a sheet shape.

(ii) A beam profile measured by a gas sheet monitor agrees with a beam profile measured by a wire scanner monitor applying the developed approximate analysis. The root mean square values of the beam distributions measured by two kinds of profile monitors are consistent. In addition, a two-dimensional beam profile mapping was successfully obtained.

(iii) Beam-loss rate increases linearly against the gas sheet flux. Profile measurements with enough accuracy are possible with less than about $0.2 \%$ beam loss.

These measurement results lead to a conclusion that the gas sheet monitor can measure a $3 \mathrm{MeV} 60 \mathrm{~mA} \mathrm{H}^{-}$beam profile in the order of $\mu \mathrm{s}$ as demonstrated in the preliminary experiment. This possibility may enable time evolution measurement of a beam spatial structure. The gas sheet monitor will serve as the system to monitor and control the beam in a future high-energy high-intensity particle accelerator.

\section{ACKNOWLEDGMENTS}

We are grateful to Y. Hikichi and the RCS vacuum group members for helping with experiments. We are also grateful to K. Hirano, Y. Kondo, T. Morishita, T. Miyao, and the rf group members for helping with experiments and giving useful advice. We would like to thank the ion source group members for maintaining and conditioning the beam.

[1] A. Miura, K. Hasegawa, T. Miyao, T. Maruta, Y. Liu, and K. Horioka, Beam profile monitor for intense, negative hydrogen-ion beams in the J-PARC Linac, J. Korean Phys. Soc. 69, 1005 (2016).

[2] D. Vilsmeier, M. Sapinski, and R. Singh, Space-charge distortion of transverse profiles measured by electronbased ionization profile monitors and correction methods, Phys. Rev. Accel. Beams 22, 052801 (2019).

[3] B. Hochadel, F. Albrecht, M. Grieser, D. Habs, D. Schwalm, E. Szmola, and A. Wolf, A residual-gas ionization beam profile monitor for the Heidelberg test storage ring TSR, Nucl. Instrum. Methods Phys. Res., Sect. A 343, 401 (1994).

[4] H. Harada, K. Yamamoto, and M. Yoshimoto, in Proceedings of the 3rd International Particle Accelerator Conference, New Orleans, LA, 2012 (IEEE, Piscataway, NJ, 2012), MOPPR029.

[5] D. A. Bartkoski, C. Deibele, and Y. Polsky, Design of an ionization profile monitor for SNS accumulator ring, Nucl. Instrum. Methods Phys. Res., Sect. A 767, 379 (2014).

[6] T. Tsang, D. Gassner, and M. Minty, Residual gas fluorescence monitor for relativistic heavy ions at RHIC, Phys. Rev. Accel. Beams 16, 102802 (2013).
[7] F. Becker, C. Andre, P. Forck, and D. H. H. Hoffmann, in the 8th European Workshop on Beam Diagnostics and Instrumentation for Particle Accelerators (DIAPAC), Venice, Italy (JACoW, 2017), MOO3A02.

[8] C. Andre, P. Forck, R. Haseitl, A. Reiter, R. Singh, and B. Walasek-Hoehne, in Proceedings of the 3rd the International Beam Instrumentation Conference 2014, Monterey, CA (JACoW, 2014), TUPD05.

[9] M. U. Bug, E. Gargioni, H. Nettelbeck, W. Y. Baek, G. Hilgers, A. B. Rosenfeld, and H. Rabus, Ionization cross section data of nitrogen, methane, and propane for light ions and electrons and their suitability for use in track structure simulation, Phys. Rev. E 88, 043308 (2013).

[10] M. A. Plum, E. Bravin, J. Bosser, and R. Maccaferri, $\mathrm{N}_{2}$ and $\mathrm{Xe}$ gas scintillation cross-section, spectrum, and lifetime measurements from $50 \mathrm{MeV}$ to $25 \mathrm{GeV}$ at the CERN PS and booster, Nucl. Instrum. Methods Phys. Res., Sect. A 492, 74 (2002).

[11] H. D. Zhang, E. Martin, V. Tzoganis, C. P. Welsch, N. S. Chritin, E. B. Diaz, O. R. Jones, G. Schneider, R. Veness, P. Forck, and S. Udrea, in Proceedings of the International Particle Accelerator Conference 2017, Copenhagen, Denmark (JACoW, 2017), MOPAB139.

[12] H. D. Zhang, A. Salehilashkajani, C. P. Welsch, M. Ady, J. Glutting, O. R. Jones, T. Marriott-Dodington, S. Mazzoni, A. Rossi, G. Schneider, R. Veness, P. Forck, and S. Udrea, in Proceedings of the International Particle Accelerator Conference 2019, Melbourne, Australia (JACoW, 2019), WEPGW096.

[13] V. Tzoganis, H. D. Zhang, A. Jeff, and C. P. Welsch, Design and first operation of a supersonic gas jet based beam profile monitor, Phys. Rev. Accel. Beams 20, 062801 (2017).

[14] Y. Hashimoto, T. Fujisawa, T. Morimoto, Y. Fujita, T. Honma, S. Muto, K. Noda, Y. Sato, and S. Yamada, Oxygen gas-sheet beam profile monitor for the synchrotron and storage ring, Nucl. Instrum. Methods Phys. Res., Sect. A 527, 289 (2004).

[15] N. Ogiwara, in Proceedings of the International Particle Accelerator Conference IPAC2016, Busan, Korea (JACoW, 2016), WEOBB03.

[16] N. Ogiwara, Y. Hori, I. Yamada, Y. Hikichi, J. Kamiya, and M. Kinsho, in Proceedings of the International Particle Accelerator Conference IPAC2019, Melbourne, Australia (JACoW, 2019), WEPGW037.

[17] I. Yamada, N. Ogiwara, J. Kamiya, M. Kinsho, and M. Wada, in Proceedings of the International Particle Accelerator Conference IPAC2019, Melbourne, Australia (JACoW, 2019), WEPGW041.

[18] J. Kamiya, N. Ogiwara, A. Miura, M. Kinsho, and Y. Hikichi, Non-destructive 2-d beam profile monitor using gas sheet in J-PARC Linac, J. Phys. Conf. Ser. 1067, 072006 (2018).

[19] J. Kamiya, K. Okabe, M. Kinsho, K. Moriya, I. Yamada, N. Ogiwara, Y. Hikichi, and K. Wada, Evaluation of 2-d transverse beam profile monitor using gas sheet at J-PARC Linac, J. Phys. Conf. Ser. 1350, 012149 (2019).

[20] H. Fujiwara, High-accurate numerical method for integral equations of the first kind under multiple-precision arithmetic, Theor. Appl. Mech. Jpn. 52, 193 (2003). 
[21] Y. Kondo et al., Beam test of a new radio frequency quadrupole linac for the Japan proton accelerator research complex, Phys. Rev. ST Accel. Beams 17, 120101 (2014).

[22] Y. Kondo, T. Morishita, and R. A. Jameson, Development of a radio frequency quadrupole linac implemented with the equipartitioning beam dynamics scheme, Phys. Rev. Accel. Beams 22, 120101 (2019).

[23] Molflow+, http://molflow.we.cern.ch.

[24] Nikon Corporation, http://www.nikon.com.
[25] Hamamatsu Photonics k. k., http://www.hamamatsu.com/.

[26] Bitran Corporation, http://www.bitran.co.jp.

[27] W. H. DeLuca, Beam detection using residual gas ionization, IEEE Trans. Nucl. Sci. 16, 813 (1969).

[28] H. M. Xie, K. W. Gu, Y. Wei, Y. Zhang, G. Y. Zhu, L. Jing, Z. X. Li, L. L. Li, X. J. Hu, L. J. Mao, Z. Du, and J. X. Wu, A noninvasive ionization profile monitor for transverse beam cooling and orbit oscillation study in hirfl-csr, Nucl. Sci. Tech. 31, 40 (2020). 